Wensinck, F. \& Boevé, J. J. (1957). J. gen. Microbiol. 17, 401-413

\title{
Quantitative Analysis of the Gram Reaction
}

\author{
By F. WENSINCK AND JOHANNA J. BOEVÉ \\ Medical Biological Laboratory of the National Defence Research Council T.N.O., \\ Rijsteijk-ZH, Netherlands
}

SUMMARY: The crystal violet and iodine uptake by suspensions of Staphylococcus aureus and Escherichia coli was determined. It was found that equal amounts of intracellular dye-iodine complex were formed in these micro-organisms. The molar ratio dye to iodine was $1: 2$. The extractability of this complex with methanol and ethanol was compared with the relative solubility of dye-iodine precipitates. Results with $E$. coli suggest that the solvents pass freely into the cells and decolorize them after dissociation of the dye-iodine complex. Gram-positive micro-organisms may be arranged in a number of groups according to their extractability pattern with regard to the complex. These patterns suggest the presence of interaction between the cells and ethanol. This interaction may be located at the cell wall.

The elucidation of the mechanism underlying the Gram reaction seems to be complicated by a lack of quantitative information on the successive reactions leading to differentiation. The uptake of primary stain by bacterial suspensions has been studied in some detail, but the results are divergent. Moreover, the suspensions used were, with a few exceptions, killed by heat or fixatives which are likely to have affected the staining properties. The interpretation of the effects of iodine treatment and decolorizing agents is largely based on qualitative data obtained with heat-fixed smears; as appears from the careful compilation of the literature by Bartholomew \& Mittwer (1952), these data have thus far given little information on the mechanisms involved. The object of the present work was to establish essential features of Gram-positivity and Gram-negativity. Quantitative data were collected on primary stain and iodine uptake by bacterial suspensions. The effects of fixation and of decolorizer concentration on the Gram reaction were also studied. It was found that suspensions could easily be differentiated without counter-stain; this step was therefore omitted in the procedure which consisted in staining with crystal violet, treatment with iodine and differentiation with ethanol.

\section{METHODS}

Organisms. The strains used included the Gram-positive species Staphylococcus aureus, Streptococcus faecalis, S. lactis, Diplococcus pneumoniae type II, Lactobacillus arabinosus, L. plantarum, Bacillus cereus, B. subtilis, Clostridium welchii type A, Saccharomyces cerevisiae; Gram-negative organisms included Escherichia coli, Aerobacter aerogenes, Paracolobactrum sp., Pseudomonas fuorescens, Salmonella enteritidis, Serratia marcescens.

Culture media. Staphylococcus aureus, Streptococcus faecalis, S. lactis, Bacillus cereus, $B$. subtilis and the Gram-negative rods were grown in a medium 
containing $1 \%(\mathrm{w} / \mathrm{v})$ Difco peptone, $\mathbf{0 . 3} \%$ Difco meat extract, $0.5 \% \mathrm{NaCl}$ and $1 \%$ glucose. Shake cultures were incubated for $16 \mathrm{hr}$. at $32^{\circ}$. Diplococcus pneumoniae was cultivated for $16 \mathrm{hr}$. at $37^{\circ}$ in beef heart infusion broth containing $1 \%$ Difco peptone, $0.5 \% \mathrm{NaCl}$ and $0 \cdot 1 \%$ glucose. Clostridium welchii was grown in a cooked-meat medium prepared from minced horse meat; cultures were incubated for $16 \mathrm{hr}$. at $37^{\circ}$. Stephenson \& Rowatt medium (1947) was used for lactobacilli, which were cultivated for $48 \mathrm{hr}$. at $25^{\circ}$.

Bacterial suspensions. After incubation, organisms were centrifuged down, washed with distilled water and resuspended in distilled water. Saccharomyces cerevisiae suspensions were prepared from fresh commercial baker's yeast. The final aqueous suspensions were adjusted to a concentration of $c .8 \cdot 5 \mathrm{mg}$. dry wt./ml. with the aid of a nephelometer (Evans Electroselenium Ltd.; EEL) and dry weight/turbidity reference curves. The suspensions were adjusted to $\mathrm{pH} 7 \cdot \mathbf{1 - 7 \cdot 2}$, unless otherwise stated.

Dry weight and total nitrogen determinations. Dry weights were obtained by drying $1 \mathrm{ml}$. samples of the suspensions at $110^{\circ}$ to constant weight. Total $\mathbf{N}$ determinations were performed by the Kjeldahl technique.

Gram reaction. Crystal violet (Gurr, Michrome brand, purity c. $85 \%$ ) was used as the primary stain. The solution in distilled water contained $0.96 \mathrm{mg}$. dye $/ \mathrm{ml}$., corresponding to $0.82 \mathrm{mg}$. crystal violet and $0.745 \mathrm{mg}$. crystal violet cation; stock solutions were adjusted to $\mathrm{pH} 7 \cdot \mathbf{1}$. Gram's iodine was prepared by dissolving $1 \mathrm{~g}$. iodine and $2 \mathrm{~g}$. potassium iodide in a small volume of distilled water; the resulting solution was diluted to a final volume of $300 \mathrm{ml}$. In the routine Gram-reaction procedure with bacterial suspensions, $96 \%(v / v)$ ethanol in water was used for differentiation.

The primary stain uptake was determined as follows. Two ml. of the crystal violet solution were added to different amounts of bacterial suspension to a total volume of $5 \mathrm{ml}$. The mixtures were left for $10 \mathrm{~min}$. at room temperature and centrifuged at $3000 \mathrm{rev} . / \mathrm{min}$. for $10 \mathrm{~min}$. The dye concentration of the supernatant fluid was estimated by measuring the extinction of suitable dilutions in $2 \%(\mathrm{w} / \mathrm{v})$ of dipotassium phosphate by a Gallenkamp direct reading photo-electric colorimeter with an Ilford 625 filter and comparing with a calibration curve of the dye solution. The dye uptake of the organisms was computed from the difference in dye concentration between the original staining solution and the supernatant fluid. For the determination of the retention of absorbed dye, the stained sediments were suspended in $5 \mathrm{ml}$. of distilled water or the $96 \%(\mathrm{v} / \mathrm{v})$ ethanol solution. One wash with water and three washes with the ethanol solution were sufficient, since further washing did not remove any more dye. The sediments after washing with water were resuspended in $3 \mathrm{ml}$. distilled water and mixed with $2 \mathrm{ml}$. iodine solution. The mixtures were left for $10 \mathrm{~min}$. at room temperature and centrifuged. The sediments were washed three times with $5 \mathrm{ml}$. of the ethanol solution; the dye concentrations in the ethanol washes were determined after addition of one drop of $0 \cdot 1 \mathrm{~N}-\mathrm{Na}_{2} \mathrm{~S}_{2} \mathrm{O}_{3}$ solution to the dilution of supernatant fluid in phosphate solution in order to reduce iodine. In a small series of comparative experiments absolute methanol instead of ethanol was used for differentiation. 
Analysis of crystal violet-iodine complexes. The composition of precipitates resulting from the interaction of crystal violet and iodine solutions was determined by measuring crystal violet and iodine concentrations in the supernatant fluid obtained by centrifugation of the mixtures.

Graded amounts of the iodine solution used in the Gram reaction were added to a series of centrifuge tubes containing $2 \mathrm{ml}$. crystal violet solution $(\sim 1 \cdot 49 \mathrm{mg}$. dye-cation); the total volume was $5 \mathrm{ml}$. The tubes were left at room temperature for $10 \mathrm{~min}$. and centrifuged for $10 \mathrm{~min}$. at $6000 \mathrm{rev} . / \mathrm{min}$. The supernatant fluid was analysed for crystal violet in the usual way; free iodine was titrated with $0.01 \mathrm{~N}-\mathrm{Na}_{2} \mathrm{~S}_{2} \mathrm{O}_{3}$ solution.

Iodine uptake by bacterial suspensions stained with crystal violet. To a series of centrifuge tubes containing $1 \mathrm{ml}$. bacterial suspension graded amounts of Gram's iodine solution were added. Free iodine in the supernatant fluids obtained by centrifugation for $10 \mathrm{~min}$. at $6000 \mathrm{rev}$. $/ \mathrm{min}$. was titrated with a $0.01 \mathrm{~N}-\mathrm{Na}_{2} \mathrm{~S}_{2} \mathrm{O}_{3}$ solution. The same procedure was applied to suspensions (1 ml.) stained with crystal violet and washed with distilled water. The amount of crystal violet taken up by the suspensions was varied by using crystal violet solutions of different strength. The values for free iodine at various concentrations of iodine in excess obtained with stained and control suspensions were plotted against amounts of iodine added to the suspensions. From the resulting curves the difference between iodine uptake by stained and control suspensions was calculated.

Effect of ethanol concentration on the retention of crystal violet and crystal violet-iodine complexes. The retention of crystal violet was determined by washing stained sediments three times with $5 \mathrm{ml}$. of $10-100 \%$ (v/v) ethanol solutions in water and analysing the supernatant fluids for crystal violet. For the determination of the retention of the complex between crystal violet and iodine, the gram reaction as outlined before was performed in a series of centrifuge tubes up to the decolorizing step. The washed sediments were resuspended in $5 \mathrm{ml}$. of $50-100 \%(\mathrm{v} / \mathrm{v})$ ethanol in water; three washes with the ethanol solution were made. The dye concentration in the supernatant fluids was determined in the usual way; $\mathbf{1}$ drop of a $0 \cdot 1 \mathrm{~N}-\mathrm{Na}_{2} \mathrm{~S}_{2} \mathrm{O}_{3}$ solution was added to the dilution in dipotassium phosphate solution.

Relative solubility of the crystal violet-iodine complex in alcohol. The extractability of the crystal violet-iodine complex from bacteria was compared with the relative solubility of this complex in 50-100\% (v/v) ethanol or methanol solutions in water. Quantities of crystal violet-iodine precipitate, approximately equal to the amount of dye-iodine complex formed in Gram-stained suspensions and containing $1.49 \mathrm{mg}$. dye-cation, were washed with distilled water and resuspended in $5 \mathrm{ml}$. portions of $50-100 \%(\mathrm{v} / \mathrm{v})$ alcohol in water solutions; washing was repeated three times and the amounts of crystal violet dissolved were determined as indicated before. The solubility figures thus obtained would not necessarily agree with those resulting from standard procedures. 


\section{RESULTS}

\section{Influence of $\mathrm{pH}$ value on crystal violet uptake}

Fig. 1 illustrates the results obtained with Staphylococcus aureus and Escherichia coli suspensions and crystal violet solutions adjusted at $\mathrm{pH}$ values varying from 2 to 9 . The crystal violet uptake by both $S$. aureus and $E$. coli suspensions was greatly influenced by the $\mathrm{pH}$ value and reached a maximum at $\mathrm{pH} \mathrm{7-8.} \mathrm{Suspensions} \mathrm{and} \mathrm{crystal} \mathrm{violet} \mathrm{solutions} \mathrm{used} \mathrm{in} \mathrm{the} \mathrm{Gram} \mathrm{reaction}$ were henceforth adjusted to $\mathrm{pH} 7 \cdot \mathbf{1 - 7 \cdot 2}$.

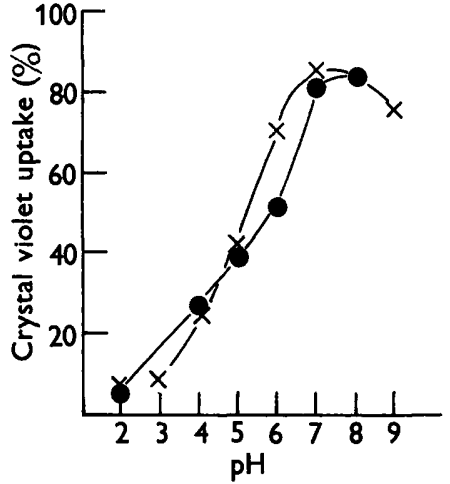

Fig. 1

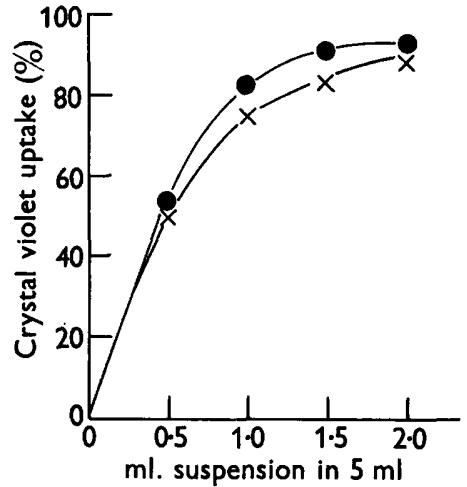

Fig. 2

Fig. 1. Influence of $\mathrm{pH}$ value on the crystal violet uptake by suspensions of Staphylococcus aureus $(\circlearrowleft)$ and Escherichia coli $(\times)$.

Fig. 2. Effect of the concentration of organisms on the crystal violet uptake by suspensions of Staphylococcus aureus (O) and Escherichia coli $(\times)$.

\section{Maximum of crystal violet uptake}

When a bacterial suspension was added to a given volume $(2 \mathrm{ml}$.) of a crystal violet solution, the crystal violet uptake increased with the amount of suspension used; it reached a maximum of approximately $90 \%$ (Fig. 2). The results presented in Fig. 2 are mean values obtained with 12 Staphylococcus aureus and Escherichia coli suspensions. They show a striking similarity between a Gram-positive and a Gram-negative organism in the pattern of crystal violet uptake. The crystal violet uptake by $0.5 \mathrm{ml}$. of the suspensions was not increased by doubling or tripling the strength of the crystal violet solution. Data concerning the maximal crystal violet uptake are summarized in Table 1. It is concluded from these data that $S$. aureus and $E$. coli absorb comparable amounts of crystal violet at $\mathbf{p H ~} 7 \cdot \mathbf{1}$.

\section{Retention of crystal violet}

On washing the stained organisms with distilled water only small amounts of crystal violet were found in the supernatant fluid after centrifugation; with $96 \%(v / v)$ ethanol in water, however, the bulk of dye was extracted (Table 2). It will be noted that the ethanol solutions decolorized Gram-positive and Gram-negative cells to the same extent. 
Table 1. Crystal violet uptake by suspensions of Staphylococcus aureus and Escherichia coli

\begin{tabular}{|c|c|c|}
\hline & S. aureus & E. coli \\
\hline $\begin{array}{l}\text { Dry weight (mg./ml. suspension) } \\
\text { Total N (\% of dry weight) } \\
\text { Crystal violet uptake: }\end{array}$ & $\begin{aligned} 8 \cdot 6 & \pm 0 \cdot 17 *(24) \dagger \\
11 \cdot 6 & \pm 0 \cdot 27(7)\end{aligned}$ & $\begin{aligned} 8 \cdot 7 & \pm 0 \cdot 23(24) \\
11 \cdot 7 & \pm 0 \cdot 26(6)\end{aligned}$ \\
\hline $\begin{array}{l}\text { By } 0.5 \mathrm{ml} \text {. of suspension (\% of } \\
\text { initial concentration) }\end{array}$ & $54 \pm 2 \cdot 4(12)$ & $50 \pm 3 \cdot 7(12)$ \\
\hline mg. dye cation/mg. dry weight & $0 \cdot 19$ & $0 \cdot 17$ \\
\hline $\mathrm{mg}$. dye cation $/ \mathrm{mg}$. $\mathrm{N}$ & $1 \cdot 64$ & $1 \cdot 45$ \\
\hline
\end{tabular}

Effect of iodine treatment on crystal violet retention

Suspensions of Staphylococcus aureus and Escherichia coli were subjected to the Gram procedure up to the stage of treatment with the counter stain and omitting, as usual, the fixation step. The results are summarized in Table 3. They clearly indicate that a Gram differentiation can be obtained with unfixed bacterial suspensions.

Table 2. Crystal violet removal from Gram-positive and Gram-negative micro-organisms by water or $96 \%(v / v)$ ethanol in water

$\begin{array}{cccc}\text { Staphylococcus aureus } & \begin{array}{c}\text { Crystal violet } \\ \text { taken up } \\ (\mathbf{m g} .)\end{array} & \overbrace{\text { Water }}^{\text {Crystal violet removed (\%) }} & \text { Ethanol } \\ \text { other positive organisms } \dagger & 1 \cdot 22 & 7(15)^{*} & 82(8) \\ \text { Escherichia coli } & 1 \cdot 26 & 10(9) & 87(5) \\ \text { other negative organisms } & 1 \cdot 22 & 12(16) & 85(8) \\ & 1 \cdot 16 & 14(10) & 88(6)\end{array}$

* Number of determinations.

$\dagger$ Viz. Streptococcus faecalis, Diplococcus pneumoniae, Lactobacillus plantarum, Bacillus subtilis and Clostridium welchii.

‡ Viz. Paracolobactrum sp., Aerobacter aerogenes, Salmonella enteritidis, Pseudomonas fluorescens and Serratia marcescens.

The residual colour of the suspensions corresponded to that of heat-fixed smears stained by the usual Gram procedure (counter-staining omitted). From Tables 2 and 3 it is apparent that the iodine treatment is essential for differentiation.

In a small series of experiments to compare the decolorization by different alcohols, iodine-treated suspensions were washed with ethanol or methanol solutions. The results listed in Table 4 show that methanol decolorizes Grampositive as well as Gram-negative organisms. This effect could not be attributed to a difference in the solubility of the crystal violet-iodine complex in these alcohols. As appears from Fig. 5, the complex dissolves more readily in ethanol than in methanol solutions. 


\section{Analysis of crystal violet-iodine complexes}

On addition of iodine to crystal violet solutions a flocculent precipitate appears which can easily be removed by centrifugation; in the presence of an excess of iodine, however, a rather stable suspension is formed of small dye particles which are difficult to spin down. The supernatant fluids obtained on centrifugation of crystal violet-iodine mixtures prepared by adding varying quantities of Gram's iodine solution (3.18 mg. iodine $/ \mathrm{ml}$.) to constant volumes

Table 3. Crystal violet removal from suspensions of Staphylococcus aureus and Escherichia coli treated with iodine and washed with $96 \%(v / v)$ ethanol in water

$\begin{array}{lcc} & \begin{array}{c}\text { Crystal violet } \\ \text { taken up (mg.) }\end{array} & \begin{array}{c}\text { Crystal violet } \\ \text { removed (\%) }\end{array} \\ \text { S. aureus (25)* } & 1 \cdot 18 & 40 \\ \text { E coli }(\mathbf{2 5}) & 1 \cdot 21 & \mathbf{9 7}\end{array}$

* Number of determinations.

Table 4. Crystal violet removal from suspensions treated with iodine and washed with methanol or $96 \%$ (v/v) ethanol in water

$\begin{array}{lccc} & \begin{array}{l}\text { Crystal violet } \\ \text { taken up (mg.) }\end{array} & \overbrace{\text { Methanol }}^{\text {Crystal violet removed (\%) }} & \text { Ethanol } \\ \text { Staphylococcus aureus }(3) * & 1.00 & 100 & 12 \\ \text { Bacillus subtilis (2) } & 1 \cdot 22 & 75 & 35 \\ \text { B. cereus (2) } & 1.25 & 82 & 29 \\ \text { Escherichia coli (3) } & 1.06 & 100 & 97 \\ & * \text { Number of determinations. }\end{array}$

of a crystal violet solution $(2 \mathrm{ml}$.) were analysed for crystal violet and free iodine. The results are indicated in Fig. 3, which shows that crystal violet was completely precipitated by $0.325 \mathrm{ml}$. iodine solution. The crystal violet solution contained $1.49 \mathrm{mg}$. dye cation which was precipitated by $0.325 \times 3.18 \mathrm{mg} .=1.03 \mathrm{mg}$. iodine. The weight to weight ratio of crystal violet to iodine in the precipitate $(1 \cdot 49: 1 \cdot 03=1 \cdot 44)$ indicates equimolarity. Thus, at the iodine concentration indicated the precipitate consists of equal numbers of crystal violet cations and iodine molecules. However, as may be seen from the results in Fig. 3, the iodine concentration could be doubled without iodine appearing in the supernatant fluid. The ratio of crystal violet cations to iodine molecules in the precipitate at this high iodine concentration is therefore 1:2. It thus appears that two species of crystal violet-iodine complexes are formed, their abundance in any precipitate depending on the ratio between the concentrations of dye and iodine in the original mixture.

\section{Crystal violet-iodine complex in bacteria}

Determinations of iodine uptake showed that more iodine is taken up by stained than by unstained bacteria. By varying the amount of dye absorbed by the suspension a relationship between extra iodine uptake and dye ab- 
sorbed could be demonstrated. This is shown in Fig. 4 which illustrates results obtained with Staphylococcus aureus and Escherichia coli. The mean ratio of crystal violet:extra iodine was 0.76 ; this value corresponds to one crystal violet-cation for two iodine molecules taken up, and agrees well with the dyeiodine proportion occurring in precipitates formed in the presence of an excess of iodine. It is clear from these results that iodine treatment of stained suspensions of a Gram-positive and a Gram-negative organism results in the formation of presumably identical dye-iodine complexes.

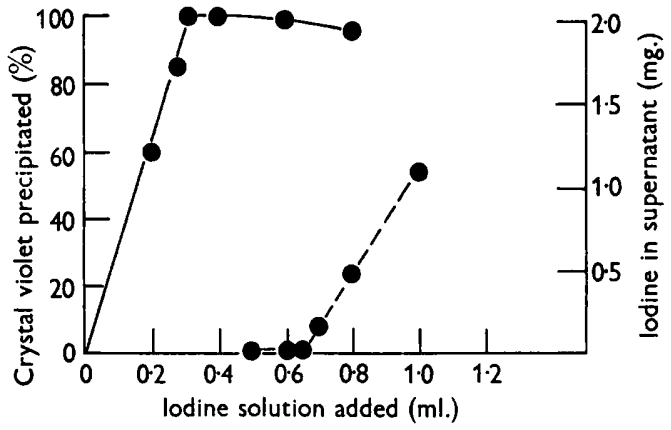

Fig. 3

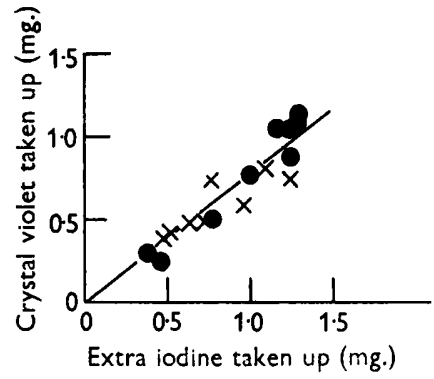

Fig. 4

Fig. 3. Crystal violet in precipitates (-) and free iodine (-- ) in supernatant fluids from mixtures of crystal violet and iodine solutions. The iodine solution (3.18 mg. iodine/ml.) was added to $2 \mathrm{ml}$. crystal violet solution $(0.745 \mathrm{mg}$. crystal violet cation $/ \mathrm{ml}$.) and the mixtures centrifuged.

Fig. 4. Relation between crystal violet and extra iodine uptake by suspensions of Staphylococcus aureus (O) and Escherichia coli $(\times)$.

\section{Effect of the ethanol concentration on the extractability of the crystal violet-iodine complex}

It was concluded from the above results that the differences between the extractability of dye-iodine complexes from Gram-positive and Gram-negative organisms might be due to differences in the interaction of the cells with the solvent. Early in the literature reports were published showing that the addition of water to ethanol increases decolorization. This effect was studied in the present work. Suspensions of Gram-positive or Gram-negative organisms were stained with crystal violet and treated with iodine; the extractability of the dye-iodine complex by different concentrations of ethanol was determined in the usual way and compared with the relative solubility of dye-iodine precipitate in these solvents; this is illustrated in Fig. 5.

The mean extractability curves obtained with Escherichia coli and other Gram-negative organisms show that extraction of the dye complex by 80 to $100 \%(\mathrm{v} / \mathrm{v})$ ethanol in water is nearly complete (Fig. 6). There is a close correlation between the curves in Fig. 6 and the solubility curve of the dyeiodine precipitate(Fig. 5). This correlation suggests that ethanol passes freely in and out of these cells. Dissociation of the dye-iodine complex is followed 
by a distribution of the intracellular crystal violet over the total volume of ethanol solution, resulting in decolorization.

The results obtained with Gram-positive organisms were completely different (Fig. 7), and conformed to the solubility curve of the dye-iodine precipitate (Fig. 5) up to a certain concentration of ethanol. Beyond this critical concentration, which varies for different groups of Gram-positive organisms, a sharp decrease of extractability occurs. It is seen from the curves that organisms which are known to be strongly Gram-positive show low maxima of extractability.

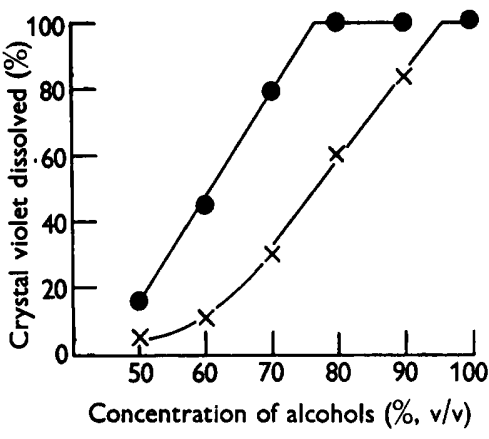

Fig. 5

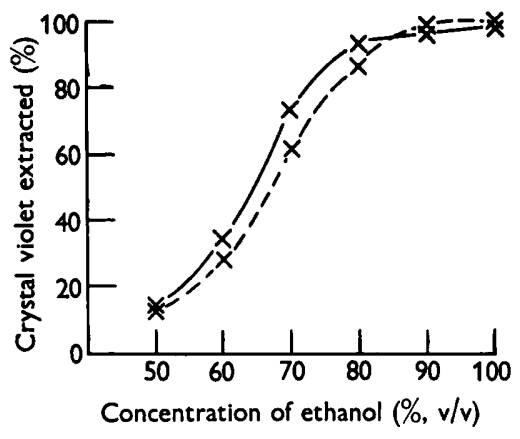

Fig. 6

Fig. 5. Relative solubility of crystal violet-iodine precipitates containing $1.49 \mathrm{mg}$. dye cation in $50-100 \%(v / v)$ ethanol $(O)$ or methanol $(x)$ in water solutions. Precipitates were prepared in the presence of an excess of iodine; ratio of crystal violet cations: iodine molecules $=1: 2$ (see text).

Fig. 6. Effect of the ethanol concentration on the extractability of the crystal violetiodine complex from Escherichia coli $(\times-\times)$ and other Gram-negative organisms $(\times---\times)$, viz. Serratia marcescens, Aerobacter aerogenes and Paracolobactrum sp.

In Fig. 8 maximum values of crystal violet extraction have been plotted against the corresponding ethanol concentrations. The distribution of the maxima along the relative solubility curve of the crystal violet-iodine precipitate suggests that the absolute value of a maximum for a given organism depends on the ethanol concentration at which it is reached. According to the strength of the ethanol solution required for maximal extraction the organisms studied may be arranged in the following sequence of Gram-positivity: yeast, spore-forming organisms, lactobacilli, micrococci, streptococci.

When the pretreatment with iodine of suspensions of Staphylococcus aureus and Escherichia coli was omitted the extraction pattern of the dye by varying concentrations of ethanol was similar for both organisms. Figure 9 shows the extractability of crystal violet and the crystal violet-iodine complex as a function of the ethanol concentration. The inability of high concentrations of ethanol to extract dye which it is suggested is a characteristic of Gram positivity, clearly depends on pretreatment with iodine. 

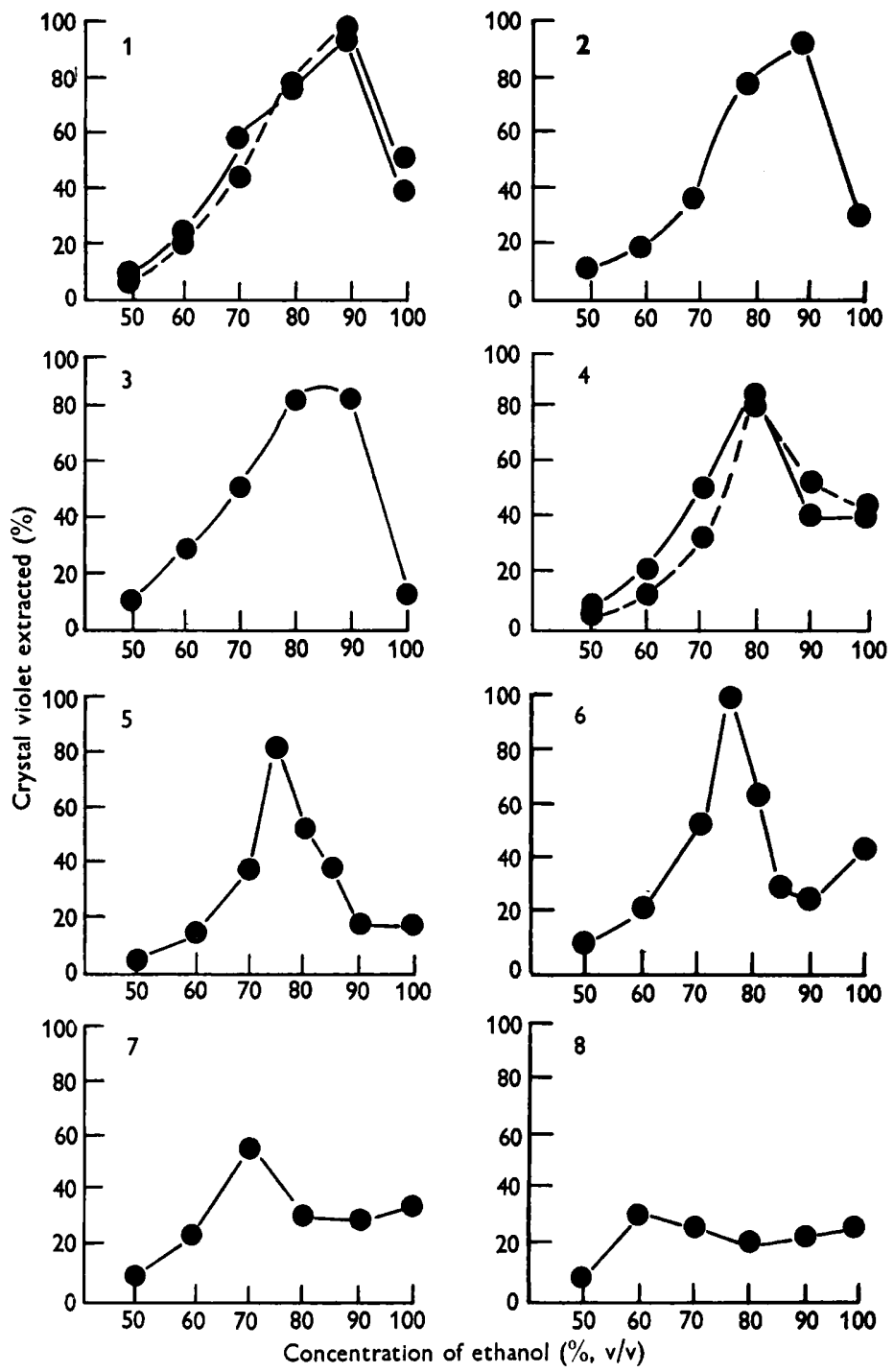

Fig. 7. Effect of ethanol concentration on the extractability of the crystal violet-iodine complex from Gram-positive micro-organisms. $1=$ Streptococcus faecalis $(-)$ and S. lactis $(---) ; 2=$ Diplococcus pneumoniae; $3=$ Staphylococcus aureus; $4=$ Lactobacillus plantarum (-); L. arabinosus $(---) ; 5=$ Clostridium reelchii ; $6=$ Bacillus cereus $; \mathbf{7}=\boldsymbol{B}$. subtilis; $8=$ Saccharomyces cerevisiae.

\section{Gram reaction in fixed suspensions}

From the above experiments it is apparent that perfect Gram differentiation may be obtained with native bacterial suspensions. It was therefore concluded that fixation plays no essential role in the Gram reaction. Nevertheless, fixation has definite effects on the primary stain uptake by suspensions. In a small series of experiments, suspensions of Staphylococcus aureus and 
Escherichia coli were heated in a boiling-water bath for periods from $30 \mathrm{~min}$. to $16 \mathrm{hr}$. This treatment, involving a considerable loss of cell material (consisting mainly of ribonucleic acid), leads to a decrease of the uptake of crystal violet. Washing with distilled water, however, restored the capacity to absorb dye of the heated organisms to that of the native suspension; Gram reactions on these washed suspensions gave normal results. The same applies to suspensions treated with $96 \%(\mathrm{v} / \mathrm{v})$ ethanol-ether $(1: 1)$, a mixture used for the fixation of slide preparations. Fig. 10 illustrates the results of these experiments.

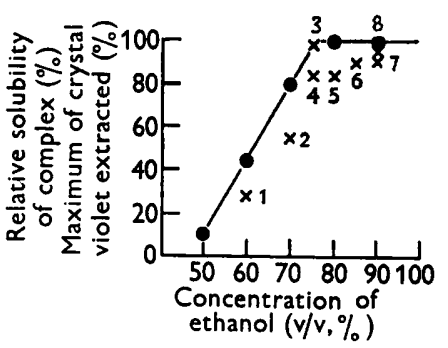

Fig. 8

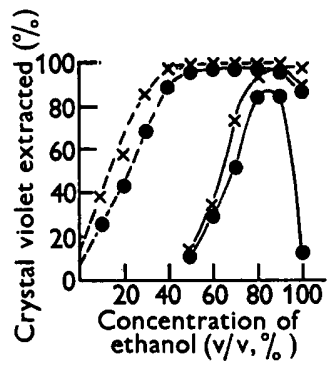

Fig. 9

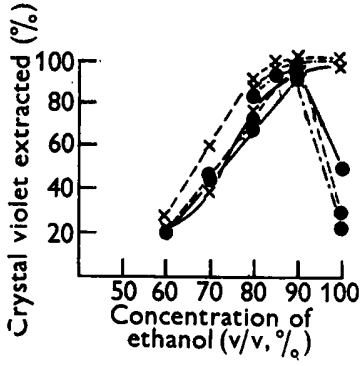

Fig. 10

Fig. 8. Diagram indicating the relationship between maximum values of crystal violet extraction and ethanol concentration. - - , relative solubility of crystal violetiodine precipitate. $1=$ Saccharomyces cerevisiae $; 2=$ Bacillus subtilis; $3=B$. cereus; 4=Clostridium welchii ; 5=Lactobacilli; $6=$ Staphylococcus aureus; $7=$ Diplococcus pneumoniae; $8=$ Streptococci.

Fig. 9. Extractability of crystal violet (-- ) and the crystal violet-iodine complex (-) from suspensions of Staphylococcus aureus $(\bullet)$ and Escherichia coli $(\times)$ by $10-100 \%$ (v/v) ethanol.

Fig. 10. Extractability of the crystal violet-iodine complex from fixed suspensions of Staphylococcus aureus $(0)$ and Escherichia coli $(\times)$. - , fixation at $100^{\circ}$ for $30 \mathrm{~min}$.; ---, fixation at $100^{\circ}$ for $60 \mathrm{~min} . ;-. .-.-$, fixation with a $96 \%$ (v/v) ethanol-ether (1:1) mixture.

\section{DISCUSSION}

The results of our experiments on crystal violet uptake (Fig. 2 and Table 1) show that equal amounts of dye are taken up by standardized native suspensions of a Gram-positive and a Gram-negative organism. The values calculated as $\mathrm{mg}$. dye cation/mg. dry weight or $\mathrm{mg}$. bacterial $\mathbf{N}$ agree well with the data of Bartholomew \& Finkelstein (1954) for heat-killed Staphylococcus aureus and Escherichia coli. From a few experiments Kennedy \& Barbaro (1953) concluded that more crystal violet was taken up by Gram-positive than by Gram-negative organisms; a Gram-variable corynebacterium assumed an intermediate position. However, the figures reported by these authors (Barbaro \& Kennedy, 1954) for the uptake of crystal violet are widely spread, according to them because of many variables. Finkelstein \& Bartholomew (1956) drew attention to the decolorizing effect of the buffer solutions used by Barbaro \& Kennedy and concluded that no correlation between Gramcharacter and crystal violet uptake exists when suspensions are washed with distilled water. As no difference in crystal violet uptake was found between 
$S$. aureus and $E$. coli at various pH values (Fig. 1) it seems probable that the Stearn \& Stearn concept (1924) which correlates Gram character with dye uptake capacity should be abandoned. This concept is essentially based on presumed differences between iso-electric points of Gram-positive and Gramnegative cells. These differences could not be demonstrated by electrophoresis (Harden \& Harris, 1953).

A simple explanation of the effect of iodine on the extractability of crystal violet from Gram-positive and Gram-negative organisms by $96 \%$ (v/v) ethanol in water (Tables 2 and 3) might be a difference in the interaction between crystal violet and iodine in the two groups. This possibility, however, seems to be ruled out by the results of experiments on iodine uptake by stained suspensions; these showed that the dye-iodine complex in both Staphylococcus aureus and Escherichia coli contains two molecules of iodine/dye cation (Fig. 4) and corresponds to crystal violet-iodine precipitates formed in the presence of excess iodine (Fig. 3). The extractability of the dye-iodine complex from Gram-negative organisms seems to be merely a function of its solubility. The curves (Fig. 6) suggest that rapid penetration of ethanol occurs. The extractability from Gram-positive organisms however parallels the solubility only up to a critical concentration of ethanol. Beyond this concentration the extractability is considerably decreased (Fig. 7). This may be due to a decrease of penetration or to interference with the outward diffusion of the solvent. These processes were studied in heat-fixed smears stained with crystal violet and treated with iodine. In $S$. aureus smears immersed in $80 \%$ $(\mathrm{v} / \mathrm{v})$ ethanol in water solution decolorization was preceded by a colour change from black to blue resulting from dissociation of the dye-iodine complex. Immersion in absolute ethanol was not followed by colour changes, indicating that no dissociation occurred. It was concluded from this observation that in Gram-positive micro-organisms the penetration is impeded by high concentrations of ethanol.

Iodine treatment is essential for the demonstration of decreased penetration of the solvent. Fig. 9 illustrates the importance of iodine treatment and ethanol concentration in the Gram-differentiation. It is seen that comparable amounts of the dye-iodine complex are extracted from Staphylococcus aureus by $50 \%(v / v)$ aqueous and absolute ethanol. It may be concluded therefore that the final intra-cellular concentration of ethanol is $c .50 \%$ in both cases. The curves show that the extractability of crystal violet in the absence of iodine is nearly complete at this concentration of ethanol. Clearly, the function of iodine is to lower the solubility of the absorbed dye to a critical level at which it is very susceptible to the concentration of the solvent. This supposition finds support in the demonstration that suitability of basic dyes for the Gram reaction depends partly on the solubility of their iodine complexes in ethanol (Bartholomew \& Mittwer, 1951). Specific effects of solvents however are important. Thus, methanol was found to decolorize both Grampositive and Gram-negative organisms (Table 4) in spite of a lower solubility of the crystal violet-iodine precipitate in this solvent than in ethanol (Fig. 5). 
The nature of the substrate in Gram-positive organisms which interacts in a characteristic way with ethanol and ethanol-water mixtures is unknown. It was concluded from experiments with fixed suspensions (Fig. 10) that this substrate is highly thermostable. Moreover, Gram-reactions produced normal results with suspensions of Staphylococcus aureus pretreated with solvents which were shown to decolorize $S$. aureus completely (methanol, $80 \%$ (v/v) ethanol in water). It is therefore concluded that solvent interactions with Gram-positive material are reversible. They are likely to occur in the cell wall, known from early experiments (Burke \& Barnes, 1929) to play a major role in the Gram reaction.

From these considerations it appears that a study of cell-wall properties and particularly of interactions with organic solvents will provide much more information on Gram-positivity than will attempts to isolate Gram-positive cell material. Crystal violet stains almost every type of cell and forms intracellular complexes with iodine which have not been shown to be associated with a particular cell component. The fact that this complex cannot easily be extracted from a small fraction of cell types may be related to the characteristic structure of their cell walls. Some of the prominent features of Grampositive cell walls are their low lipid content (Salton, 1953) and the presence of glycerophosphate which may be part of a lipid-glycerophosphate-protein cell-wall component (Mitchell \& Moyle, 1951, 1954). The effect of glycerophosphate removal on Gram-positivity which has been reported by Mitchell \& Moyle (1950) remains to be studied in detail.

\section{REFERENCES}

Barbaro, J. F. \& Kennedy, E. R. (1954). A quantitative gram reaction. J. Bact. $67,603$.

Bartholomew, J. W. \& Finkelstein, H. (1954). Crystal violet binding capacity and the gram reaction of bacterial cells. J. Bact. 67, 689 .

Bartholomew, J. W. \& MrtTwer, T. (1951). The mechanism of the Gram reaction. III. Solubility of dye-iodine precipitates and further studies on primary dye substitutes. Stain Technol. 26, 231.

Bartholomew, J. W. \& Mittwer, T. (1952). The Gram stain. Bact. Rev. 16, 1.

Burke, V. \& Barnes, M. W. (1929). The cell wall and the Gram reaction. J. Bact. $18,69$.

Finkelstein, H. \& Bartholomew, J. W. (1956). Influence of phosphate buffer on crystal violet uptake and retention by bacterial cells as related to the Gram reaction. J. Bact. 72, 340.

Harden, V. P. \& Harris, J. O. (1953). The isoelectric point of bacterial cells. J. Bact. 65, 198.

Kennedy, E. R. \& Barbaro, J. F. (1953). Quantitative adsorption of crystal violet. J. Bact. 65, 678.

Mrtchell P. \& Moyle, J. (1950). Occurrence of a phosphoric ester in certain bacteria: its relation to Gram staining and penicillin sensitivity. Nature, Lond. 166, 218.

Mitchell, P. \& Moyle, J. (1951). The glycerophospho-protein complex envelope of Micrococcus pyogenes. J. gen. Microbiol. 5, 981.

Mitchell, P. \& Moyle, J. (1954). The Gram reaction and cell composition : nucleic acids and other phosphate fractions. J. gen. Microbiol. 10, 533. 
Salton, M. R. J. (1953). Studies of the bacterial cell wall. IV. The composition of the cell wall of some Gram-positive and Gram-negative bacteria. Biochim. biophys. Acta, 10, 512.

Stearn, E. W. \& Stearn, A. E. (1924). The chemical mechanism of bacterial behaviour. I. Behaviour towards dyes; factors controlling the Gram reaction. II. A new theory of the Gram reaction. J. Bact. 9, 463, 479.

Stephenson, M. \& RowatT, E. (1947). The production of acetylcholine by a strain of Lactobacillus plantarum. J. gen. Microbiol. 1, 279.

(Received 21 March 1957) 